\title{
IPPOG Global Cosmic Rays Portal: Making Cosmic Rays Studies available to schools worldwide
}

\section{Barbora Bruant Gulejova on behalf of the IPPOG Collaboration}

(a complete list of authors can be found at the end of the proceedings)

University of Bern,

Sidlestrasse 5, Bern, Switzerland,

E-mail: barbora.gulejova@cern.ch

The International Particle Physics Group (IPPOG) is a global network active in informal education and outreach in particle physics and related research, including cosmic-ray and astro-particle physics. Since many years, IPPOG has been actively supporting the International Cosmic Day organized by DESY and the International Muon Week organized by Quarknet. In 2015 IPPOG started work on establishing a universal portal through which successful cosmic-ray study programmes can reach out to teachers and students around the world. This common web platform is being developed by IPPOG in the frame of its new IPPOG web pages. It will contain all information for schools interested in taking part in cosmic-ray experiments and analysing real data in classroom; also instructions about how to build, borrow, or purchase cosmic-ray detectors. The platform will also facilitate collaboration and exchanges between the experiments' project managers and advertising of related events. 


\section{Introduction: Challenges of the particle physics community}

The main challenges the scientific community faces currently are the threat to the financial support of large experimental endeavours and falling interest of young people to engage in studies of STEM (science, technology, engineering and mathematics), especially physics, and mistrust in science. In general physics is less popular than other natural sciences, suffering a stigma of being a science that is very mathematical, abstract, and complicated. It is perceived as disconnected from the real world. Most of the students who claim to love science do not mean physics, not realizing how much fun physics can be. It is important to break these stereotypes based on the misperception of physics in society, and lack of awareness and understanding of it by the public. Physics is a pillar of all natural sciences. Physics is about understanding the basic laws of nature, the world we live in. It explains how the world around us and within us functions. This fundamental definition of physics does not often come across to students from their physics school curriculum.

A new generation of STEM specialists must prepare innovative solutions for tomorrow, one in which both genders have an equally important role to play. Moreover, future particle physics / high-energy physics projects will require a long-term, worldwide commitment of significant monetary resources and human expertise. Today in Europe less than $20 \%$ of the young people choose STEM studies, while the number of STEM jobs grows three times faster than any other job. According to the estimates of UNESCO, there will be 7 million new European jobs in STEM by 2025 and not enough people to fill them if nothing changes [1]. A nation-wide German survey in 2000 [2] has revealed that almost $70 \%$ of the students drop physics at the earliest possible opportunity, which in some parts of the country already happens at an age of 15 to 16 years. Why is that? Let's start our analysis by stating that the only modern physics topic students are confronted with by that age is the physics of nuclear reactors.

\subsection{Rare exposure of society to modern physics}

We live in a modern world surrounded by technologies resulting from the greatest scientific discoveries of the past decades that are underpinned by modern physics of the $20^{\text {th }}$ and $21^{\text {st }}$ centuries. The cell phones and computers upon which we rely were developed thanks to a fundamental understanding of Physics. Despite this, the physics taught in most of the high school classrooms is stuck in the past. "I always found it dry and removed from the real world, but I learned that physics can be extremely fascinating and relevant, and I now realized that I actually enjoy physics, just not what is taught in our school curriculum.", claims a 16-years old participant of the programme 'Creating Ambassadors for Science in Society' [3] organized under the umbrella of the International Particle Physics Outreach Group (IPPOG) [4]. Moreover, the misinformation and disinformation by mainstream media increases even more the gap between scientists and non-scientists in perception of physics and basic research.

Almost 50 years after the Standard Model was developed, in typical curricula worldwide physics lessons often take the form of a history class with live demos and occasional hands-on experiments. This gives an impression that physics' greatest discoveries were in the past. Most of the students end up finishing high school believing that there are only 3 elementary particles (electrons, protons, neutrons) and only knowing 2 types of forces (gravitational and electromagnetic) all as classically described. They never learn that the electron has five other sibling particles - electron neutrino, muon, muon neutrino, tau, and tau neutrino - which are collectively called leptons. They never learn that everything in the universe, from the cereal they eat for breakfast to the galaxies above, is fundamentally composed of just two quarks and one lepton. They will never learn that everything has the same origin and we are all stardust. 


\subsection{Why understanding of particle physics by a broad population matters}

Exposure to modern physics such as particle physics and its technological applications increases the interest of students in physics and their perception of today's role of physics and physicists in society. The impact of different types of extra-curricular activities in particle physics (e.g. exhibitions, the physics masterclass programme [5] and teaching [6]) on students aged 1619 years in UK and Germany has been evaluated [7], clearly showing that their general interest in physics has increased very strongly. Students would like to deal more often with modern physics at school and criticized the lack of practical experiments.

Particle physics and related sciences permits the demonstration of the scientific method, to show how advanced science is, where it is going, and how it works, and points out the technological advances in those processes. It also shows students how dynamic and collaborative research is, how experimentation has become both crucial and harder to accomplish. They learn the process through which science develops theories and validates models, how experiments are designed and built in a worldwide collaborative manner in order to validate theories and models through the analysis of data from large experiments.

\subsection{Importance of outreach, informal education and extra-curriculum activities}

The importance of the efforts to broaden the spectrum of modern physics available at schools has been recently officially recognised by the full particle physics community. The European Strategy for Particle Physics Update 2020 recommended the teaching of the Standard Model in school classrooms: "The particle physics community should work with educators and relevant authorities to explore the adoption of basic knowledge of elementary particles and their interactions in the regular school curriculum" [8].

The current, well-focused concerted and global outreach and communication efforts to engage the public are already today of vital strategic importance. Until we update our school curricula, science outreach, informal education and extra-curriculum activities are the only possibility to bridge the gap between science and society and bring a better understanding of our fascinating world to schools and the public. The International Particle Physics Outreach Group (IPPOG) [4] has been making a concerted and systematic effort to present and popularise particle physics across all audiences and age groups for almost 25 years and today the scientific community has in IPPOG a strategic pillar for bringing contemporary physics and society closer, fostering longterm, sustainable support for fundamental scientific research around the world.

\section{International Particle Physics Outreach Group (IPPOG)}

IPPOG is A network of scientists, science educators and communication specialists working across the globe in science education and public outreach for particle physics. IPPOG brings new discoveries in this exciting field to young people and conveys to the public that the beauty of nature is indeed becoming understandable from the interactions of its most fundamental parts - the elementary particles. IPPOG contributes to global efforts in strengthening cultural awareness, understanding and support of particle physics and related sciences and in developing the next generation of researchers. More specifically, IPPOG's purpose is to raise standards of public outreach and science education efforts. IPPOG goals are also to establish understanding of scientific process, to instil the appreciation of fundamental research and importance of evidencebased reasoning, to build trust with communities.

IPPOG has evolved from group of friends (original European Particle Physics Outreach Group set-up in 1997) into an increasingly sustainable international collaboration with a growing 
membership and a modest budget for the development of the core infrastructure needed to support major worldwide programmes in education and outreach. Currently there are 37 IPPOG members: 30 countries, 6 experiments and CERN as an international laboratory.

The IPPOG experts come from prominent national or international professional physics centres, societies and laboratories engaged in particle physics research, and from major particle physics experiments. The diversity of their cultural and educational backgrounds brings a large and important variety of skills to the table, which permit for the effective development of novel outreach activities with maximal impact. IPPOG members represent links to several nationallevel science networks. This constitutes IPPOG's global network of laboratories, institutions, organizations and individuals all passionate about particle physics. The expertise of IPPOG's members spans all aspects of collider and non-collider research, including astroparticle physics and accelerator and detector technology.

For its members, IPPOG is a forum for exchange of information and best practices with colleagues from around the world, brainstorming platform; resource of ideas, inspirations, training and skillbuilding ground, platform providing access to programmes for schools; (for public talks etc.). It increases international exposure, enables centralised and coordinated efforts through partnerships.

For the particle physics and the scientific community IPPOG represents a key partner for promoting their scientific mission and activities globally, a platform for engaging on a global level, building partnerships within the community and across communities, and for supporting the broader scientific objectives of particle physics on global level. The particle physics community worldwide has in IPPOG a strong partner at hand when reaching out to wider society in diverse ways that are adapted for every target audience.

In here lies the role of IPPOG as a pillar of particle physics: IPPOG is capable of reaching out to large international audiences about particle physics activities in a manner that fosters long-term, sustainable support for fundamental scientific research around the world. IPPOG develops programmes and strategy to address the current and future challenges of the particle physics and the scientific community, like declining interest in STEM-related studies, lack of support of fundamental research and mistrust in science. IPPOG's scientific education and outreach activities aim to improve public understanding and appreciation of the benefits of fundamental research, to spark interest and enthusiasm among young people, and to strengthen the integration of science in society. IPPOG not only motivates, inspires, and educates our youth in the field of particle physics, but it develops personal awareness of the value of science and of the process of evidencebased decision making, regardless of age or discipline. IPPOG helps in establishment of broad public support, as well as the commitment of key stakeholders and policy makers throughout Europe and the world for the future large-scale projects of the particle physics community.

IPPOG helps to strengthen the trust in science and its method of evidence-based decision making to offer future generations a meaningful basis that generates supportive structure in their life. Without compromising established methods, IPPOG is exploring new paths to engage citizens especially the young. Reaching out to high-school students and their teachers to convey the methods and tools used in fundamental science is a strong investment in the future. While only a fraction of young students will become scientists, and fewer still will become particle physicists, all will become ambassadors for the scientific method and evidence-based decision-making. Younger audiences will be more educated and appreciative of the importance of research, and thus more suited to make informed decisions about science and scientific questions for their nations and their peoples in the future. 


\subsection{IPPOG's Activities}

IPPOG organises global activities, but also supports local activities by sharing of expertise, best practices, resources to support events and kick-start activities. Current IPPOG activities include the ever-growing and well-established International Masterclasses in Particle Physics programme [5], the outreach Resource Database [9], the Global Cosmic Rays platform for schools currently in development (see Section IPPOG Global Cosmics), support for exhibitions and activities at public events and festivals (e.g. Colours of Ostrava in Czech Republic [10], Universal Science [11]), and different topical programmes and competitions targeting young and diverse audiences (like Particles4U [12], Girls, do Physics! [13], Creating Ambassadors for Science in Society [3], Cascade competitions in UK [14] and Slovakia [15] etc.). These diverse activities allow IPPOG to bridge the gap between science education at school and modern scientific research by inspiring, motivating and educating an especially young audience, offering hands-on experience and connecting physics to real life while using cutting edge technologies.

\section{IPPOG Global Cosmics}

Global Cosmic Rays Studies are at heart of IPPOG since several years, and recently have been established as a second IPPOG's main activity, after the International Masterclasses, the flagship activity since the beginning of the IPPOG's history. "IPPOG Global Cosmics" aim is to establish the global common web-platform for all existing succesful cosmic rays studies programmes available for students and teachers around the world.

\subsection{IPPOG and astroparticle physics}

IPPOG's physics scope is broad, including not only particle physics, but also related sciences. This means, that its expertise goes beyond LHC physics and other subjects, like neutrinos, astroparticle physics, gravitational waves, heavy ions etc. Cosmic rays studies represent considerable added value for particle physics outreach, as they allow to establish the connection with real life and even curriculum in more straightforward way, compared to other more abstract subjects. Everybody sees the stars and therefore it is relatively easy for the public to grasp the idea that particles are falling on us from the cosmos. This makes cosmic rays studies more "tangible". It allows to establish the understanding, that we are all made of particles, particles are everywhere, and they are showering on the Earth all the time in form of cosmic rays. Therefore detecting and analysing these showers by schools, using the detectors and analysis tools especially designed for educational purposes but using real data, also the data measured by big projects, have a big success.

Much of our early understanding of particle physics came from the study of cosmic rays [16]. The subsequent discoveries of massive fundamental particles in addition to the lighter, stable collection in the existing models motivated physicists to develop accelerators and detectors, eventually leading to the large-scale programmes that define the field today. Fortunately, construction and operation of the instruments needed to detect and make simple measurements of cosmic rays can be straightforward and inexpensive, making them excellent tools for the classroom. Students learn basic particle physics concepts and methods, while learning how to set up experiments, operate hardware, take data and perform analysis. As a result, cosmic ray experiments, employing a variety of detector types, have been installed in classrooms around the world. Many of these have been connected together into networks, allowing students to share data and to produce scientific results in a manner similar to our much larger-scale international collaborations. 
In order to better exploit the great potential of cosmic-ray experiments for particle physics outreach, IPPOG launched an effort to create a common umbrella for these separate programmes and create a common global cosmic rays studies online portal. IPPOG has been actively supporting and promoting International Cosmic Day (ICD) [17] organised by DESY and International Muon Week [18] organised by Quarknet on yearly basis. Through the new Global Cosmics portal, IPPOG wants to reach out to even more students worldwide and give the possibility to perform the experiments and analysis during the whole year long.

\subsection{History: From idea to new global online portal}

Following the ASPERA meeting on cosmic ray detectors for education held at CERN in 2010, several high school cosmic ray project representatives expressed an interest in working together to develop a common website and data format so that teachers and students worldwide, with or without a detector, could design and conduct studies using data from one or more projects. At the April 2015 IPPOG meeting, a panel representing five of the original cosmic ray projects (COSMIX, Extreme Energy Events, HISPARC, QuarkNet and Teilchenwelt Netzwerk) presented a brief overview of their projects and discussed renewing collaboration talks. IPPOG members made a number of suggestions and expressed an interest in participating if the initiative goes forward. In the meantime, Hans Peter Beck and Marge Bardeen had discussed a possible partnership between IPPOG and APPEC with APPEC representatives, who expressed an interest for collaboration. The efforts and strategic discussion how to kick-off the project continued during the several panel discussions on Global Cosmic Rays collaboration at following IPPOG meetings.

In February 2017 a 2-days "Workshop on High School Cosmic Ray Experiments" was organised by IPPOG at the Centro Fermi in Rome, bringing together experts from a global spectrum of cosmic-ray related educational activities.. Around 25 different programmes were presented and the willingness to continue collaboration was expressed by most of them. Valuable experiences and experimental fact-sheets were shared, the information and data sharing were discussed and student's analysis of data showcased. IPPOG has demonstrated its important role as a community builder and collaborations enabler. Indeed, the aspect of bringing people together allowed IPPOG to identify those participants of the workshop who are willing to provide their experiments, results and data for educational purposes. There are several projects around the world that address young people and teachers, to give them the opportunity to explore cosmic particles. These include projects from Finland, France, Germany, Italy, Poland, Russia, Spain, Sweden, Taiwan, UK and the US. They have been preliminarily summarised at the ICD website [19]. The workshop also resulted in creating an IPPOG Global Cosmics Steering Group, the goal of which is to develop and promote worldwide activities, connecting classrooms through their cosmic ray studies (see overview of the Global Cosmics programme in [20]). The decision has been taken to build the Global Cosmic Studies Educational Platform as part of the new IPPOG website (see below) and development of IPPOG new online portfolio started soon after.

\subsection{IPPOG new website}

Since 2018, IPPOG embarked on an ambitious project to improve the user experience across the IPPOG digital portfolio (website and social media channels) and to strengthen the IPPOG brand online by creating a new website including a new Resource Database and new Global Cosmics Platform. The goal of the new design is to greatly broaden the audience type and use of the web pages and available resources. The visual impact needs to be enhanced in order to pursue IPPOG's mission and communicate its messages to its existing and new audiences, as well as new potential members, partners and sponsors. IPPOG wants the new website to become more open to students, teachers and the general public. 


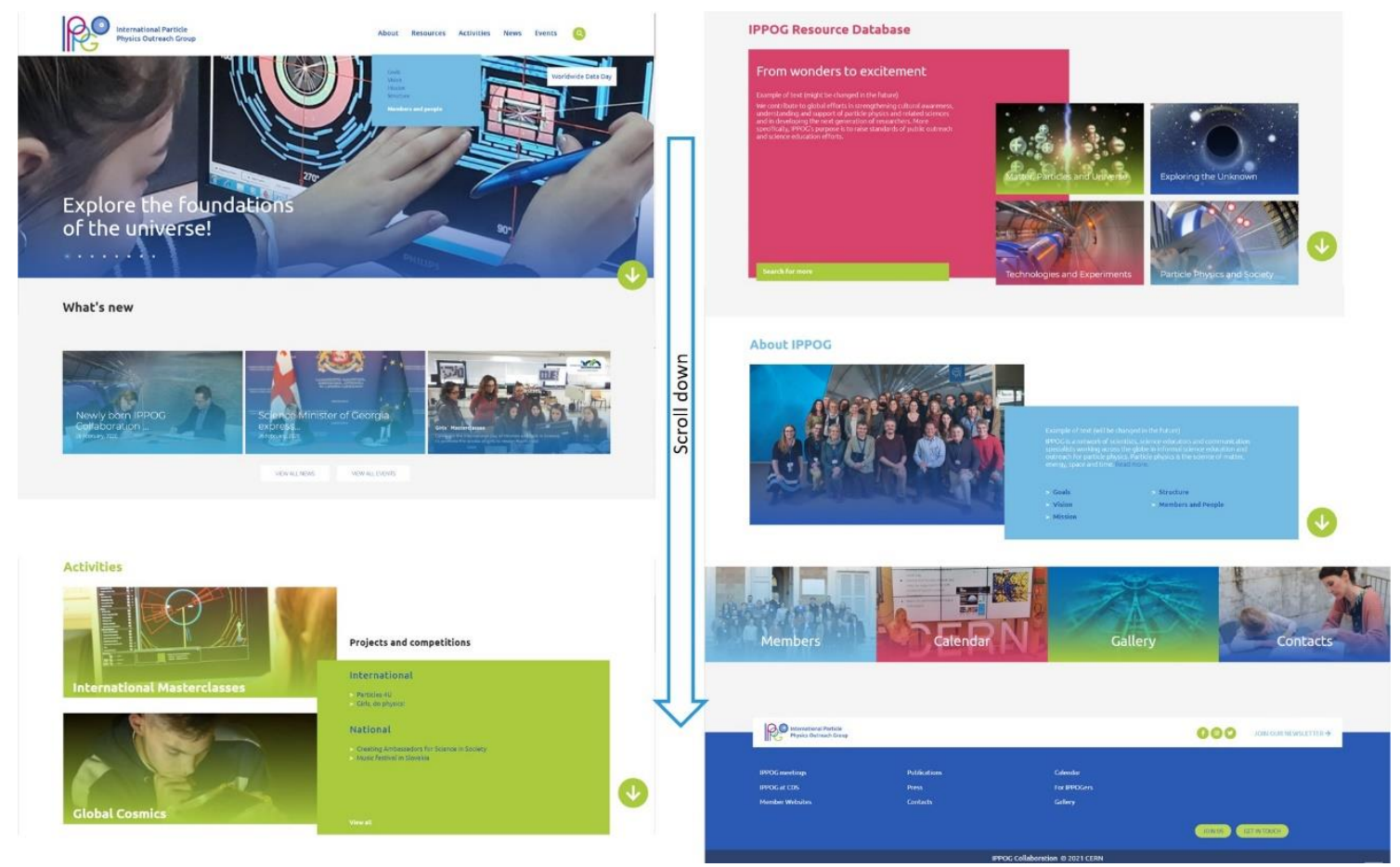

Figure 1: New IPPOG website in development. Global Cosmics Portal is accessible from the main menu on the top (submenu of "Activities") and also directly from Frontpage (see green part).

\subsubsection{IPPOG new Global Cosmic Rays Platform}

The idea is to establish a 'universal' portal through which successful cosmic ray studies programmes can reach out to teachers and students around the world. This common webpage on cosmic rays will contain information on astroparticle physics for lay audience, list of all projects, each with dedicated entry webpage with description and links, world map and list of events with possibility to request information, to join etc. The website will also offer the resources, including background information, data, analysis tools and educational framework for students' investigations. Teachers and students will learn how to build, borrow or purchase a detector for use in the classroom and translations are also envisaged. The goal is that every teacher around the world can do cosmic ray studies, get a detector for his/her school either for little money or for free and get also all the instructions and necessary guidance - through the help of IPPOG's national experts even in their language.

\subsubsection{IPPOG Resource Database}

One of the main tools IPPOG has been offering the scientific community, teachers and educators for almost ten years is the Resource Database (RDB), an online platform containing a collection of high quality engaging educational and outreach materials in particle physics and related sciences. In the last few years IPPOG has been investing considerable efforts in redesigning the $\mathrm{RDB}$ with the aim for it to become the primary source of particle physics outreach material in the world. IPPOG RDB is a collection of high quality engaging materials (e.g. videos, posters, talks, hands-on activities, tools, brochures and more) recommended by IPPOG representatives and contributors to help sharing the wonders and excitement of particle physics with teachers, students and the general public. The information available is regularly updated to reflect the latest discoveries in particle physics. Everything is freely available with the spirit of open access. The items are submitted to the database by IPPOG representatives, members of IPPOG Forum and group of trusted contributors. The IPPOG RDB is an ideal place for educational and outreach 
materials also for the astroparticle physics community, which is encouraged to submit the resources once the new website including RDB will be published.

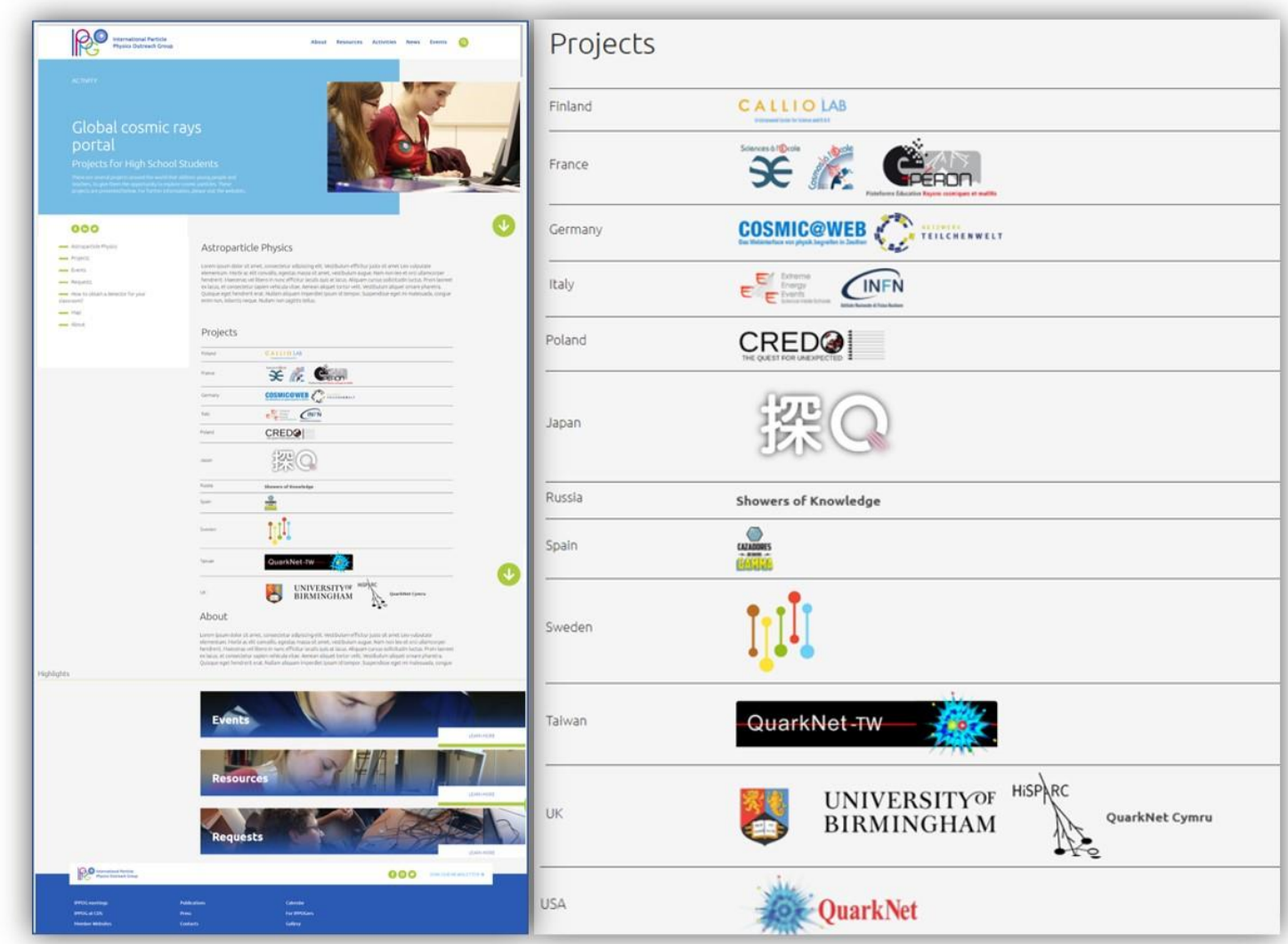

Figure 2: Global Cosmics Portal website under the development. Right: Overview of projects.

\section{References}

$\left.{ }^{1}\right]$ https://bangkok.unesco.org/content/girls-education-stem-facts

2] M.Vollmer, "Unschuldige Physik", Physikalische Blatter 56, Volume 6, Wiley-Vch,Weinheim 2000

$\left.{ }^{3}\right]$ Creating Ambassadors for Science in Society event: http://indico.cern.ch/event/736469/

$\left.{ }^{4}\right]$ International Particle Physics Outreach Group, http://ippog.org/

5] IPPOG Physics Masterclasses, http://physicsmasterclasses.org/

${ }^{6}$ ] Teaching materials (Materialien für Lehrkräfte), Netzwerk Teilchenwelt, TU Dresden, Institut für Kern- und Teilchenphysik, Dresden, Germany, https://www.teilchenwelt.de/material/materialien-fuerlehrkraefte/

$\left.{ }^{7}\right]$ M. Kobel, High school students' exposure to modern particle physics, Europhysics News Vol. 34, No. 3, 2003, pp. 108-110, https://www.europhysicsnews.org/articles/epn/abs/2003/03/epn03307/epn03307.html

$\left.{ }^{8}\right] 2020$ Update of the European Strategy for Particle Physics by the European Strategy Group, ISBN: 978-92-9083-575-2 DOI: http://dx.doi.org/10.17181/ESU2020Deliberation

${ }^{9}$ ] B.Bruant Gulejova, IPPOG - Bridging the gap between science education at school and modern scientific research, 2020, submitted to World Scientific Gribov 90, pre-print:

https://cds.cern.ch/record/2746338/files/2011.14743.pdf

${ }^{10}$ ] Music Festival in Czech Republic, Colours of Ostrava, https://www.colours.cz/

${ }^{11}$ ] Universal Science Festival, https://universalscience.web.cern.ch/

${ }^{12}$ ] Particles4U, IPPOG competition 2018, http://ippog.org/particles4u 
${ }^{13}$ ] Girls, do physics!, IPPOG campaign and competition, https://ippogorg.wixsite.com/girlsdophysics

${ }^{14}$ ] Cascade Competitions UK, https://www.birmingham.ac.uk/schools/physics/outreach/secondaryschools/cascade.aspx

${ }^{15}$ ] Cascade Competitions Slovakia, https://fyzika.uniza.sk/cascade/

${ }^{16}$ ] D. Pacini (1912). "La radiazione penetrante alla superficie ed in seno alle acque". Il Nuovo Cimento. 3 (1): 93-100: https://arxiv.org/abs/1002.1810

$\left.{ }^{17}\right]$ International Cosmic Day, DESY: https://icd.desy.de

${ }^{18}$ ] International Muon Week, QuarkNet: https://quarknet.org/content/international-muon-week

${ }^{19}$ ] Temporary collection of cosmic rays studies: https://icd.desy.de/e49245/

${ }^{20}$ ] N. Arnaud, "Global Cosmics”, proceedings of ICHEP 2020, https://indico.cern.ch/event/868940/contributions/3814079/

\section{Full Authors List: International Particle Physics Outreach Group}

Note: IPPOG Collaboration members are countries, international experiments and international laboratories. The decisionmaking body, Collaboration Board is composed of the representatives of IPPOG members. IPPOG Coordination Team comprises IPPOG co-chairs, IPPOG Staff, and Projects Coordinators. Both IPPOG Coordination Team and IPPOG Collaboration Board are listed below in alphabetical order. In the brackets after the names are not the affiliations, but the IPPOG member the person is representing or his/her function in IPPOG. Not listed are the members of IPPOG Forum, which is composed of experts contributing to IPPOG in different ways - please, see https://ippog.org for up-to-date list.

Ana Godinho (CERN), Alberto Ruiz Jimeno (Spain), Alexander Sharmazanashvili (Georgia), Andrej Gorišek (Slovenia), Anita Bens (IPPOG Coordination team), Barbora Bruant Gulejova (IPPOG Strategic Development Lead), Bolek Pietrzyk (LHCb), Carolin Schwerdt (IPPOG Global Cosmics Coordinator), Catia Peduto (Italy), Charles Timmermans (Netherlands), Christian KleinBösing (Germany), Darren Price (United Kingdom), Despina Hatzifotiadou (ALICE), Dezső Horváth (Hungary), Dirk Ryckbosch (Belgium), Eduh Duchovni (Israel), Farid Ould-Saada (Norway), Freya Blekman (CMS), Gabriel Stoicea (Romania), Ian Bearden (Denmark), Ivan Melo (Slovakia), Ivana Lagator (Montenegro), Jonas Strandberg (Sweden), Jonivar Skullerud (Ireland), Jose Ruben Alfaro Molina (HAWK), Katharina Müller (Switzerland), Ken Cecire (IPPOG International Masterclasses Coordinator), Krzysztof Wozniak (Poland), Marcelo Munhoz (Brazil), Natascha Hoermann (Austria), Nicholas Tracas (Greece), Nicolas Arnaud (France), Paul Jackson (Australia), Pedro Abreu (IPPOG Co-chair), Ralf Averbeck (GSI), Ricardo Goncalo (Portugal), Roumyana Mileva Hadjiiska (Bulgaria), Sabine Hemmer (IPPOG Global Cosmics Coordinator), Sahal Yacoob (South Africa), Sami Lehti (Finland), Sascha Mehlhase (ATLAS), Spencer Pasero (USA), Steven Goldfarb (IPPOG Co-chair), Thomas Naumann (DESY), Uta Bilow (IPPOG International Masterclasses Coordinator), Vojtech Pleskot (Czech Republic), Zdenek Dolezal (Belle II) 